\title{
Information and The Brukner-Zeilinger Interpretation of Quantum Mechanics: A Critical Investigation
}

\author{
A. Shafiee*(1), F. Safinejad ${ }^{(2)}$ and F. Naqsh ${ }^{(3)}$ \\ 1) Department of Chemistry, Sharif University of Technology, \\ P.O.Box 11365-9516, Tehran, Iran \\ 2) Department of Chemistry, Tarbiat Modarres University, \\ P.O.Box 14115-175, Tehran, Iran. \\ 3) Department of Chemistry, Kashan University, \\ Kashan, 87317-51167, Iran.
}

May 27, 2022

\begin{abstract}
In Brukner and Zeilinger's interpretation of quantum mechanics, information is introduced as the most fundamental notion and the finiteness of information is considered as an essential feature of quantum systems. They also define a new measure of information which is inherently different from the Shannon information and try to show that the latter is not useful in defining the information content in a quantum object.

Here, we show that there are serious problems in their approach which make their efforts unsatisfactory. The finiteness of information does not explain how objective results appear in experiments and what an instantaneous change in the so-called information vector (or catalog of knowledge) really means during the measurement. On the other hand, Brukner and Zeilinger's definition of a new measure of information may lose its significance, when the spin measurement of an elementary system is treated realistically. Hence, the sum of the individual measures of information may not be a conserved value in real experiments.
\end{abstract}

\section{Introduction}

Brukner and Zeilinger in a series of papers represent a new information-based interpretation of quantum mechanics which its foundation is established on

${ }^{*}$ E-mail: shafiee@sharif.edu 
the informative propositions [1-8]. They emphasize that information is the most fundamental notion in quantum mechanics and the other elementary concepts like randomness and entanglement are secondary in quantum mechanics, because all of these concepts can be deduced from definite rules of information.

Brukner and Zeilinger also believe that Shannon information ${ }^{1}$ [9] cannot be considered as an adequate measure of uncertainty for quantum measurements, since it can be derived considering the classical requirements. Unlike Shannon's approach, they do not think about any communication channel for transmission of information; rather their discussion concentrates on the information content of an isolated system. Correspondingly, they define a measure of information which its value depends on the experimental context. Yet, the total information is a major concept in their interpretation which is defined as the total knowledge that an experimentalist possesses before a complete set of mutually complementary experiments are performed $[2,4]$. In contrast to the Shannon information, the total information of a quantum system is invariant under the change of a transformation from one complete set of complementary variables to another. This means that the total information does not change when one observes the quantum phenomena in different ways [4].

The new measure of information introduced by Brukner and Zeilinger, can be applied to describe the information content of a quantum system in various situations. The entanglement swapping procedure indicated in [7] is an example. In some way, it is used to express the necessary and sufficient conditions for violation of a Bell inequality in an information-theoretical language. Other authors used the measure in the context of state estimation [10] or quantum random access codes [11].

Nevertheless, some critiques have also been appeared in different papers [12-16]. The main purpose in all these works is to show that Shannon entropy is an adequate and consistent measure of information in both the classical and quantum regimes (in the latter case, in the form of von Neumann entropy). So, some persons believe that there is no need to define an alternative measure of information. But, these engaging criticisms do not explain why the Brukner-Zeilinger measure of information cannot be regarded as a proper definition in quantum domain. Furthermore, to the present authors' knowledge, it has been remarked up to now no concrete criticism on the very conceptual basis of their interpretation.

In Brukner and Zeilinger's interpretation, two mutually connected themes can be identified. The first is based on this attitude that quantum systems carry finite information and that the finiteness of information is a fundamental principle in Nature which is inherently irreducible. So, it is expected

\footnotetext{
${ }^{1}$ We use the statements like Shannon information, Shannon's measure of information, Shannon's measure of uncertainty or Shannon entropy interchangeably here.
} 
that anything in the quantum domain can be either extracted from or explained by the notion of information. The other theme is that any quantum event can be described by a specific definition of a measure of information. This allows one to represent the information content of a quantum system via a vector in the space of information which changes with a corresponding change of the experimental setup, but its length is conserved. This means in turn that the total information content of a quantum system is conserved and does not change under a transformation in the information space.

In this paper we are going to analyze the key issues of Brukner and Zeilinger's interpretation of quantum mechanics. In section 2, the basic elements of their interpretation are reviewed. Then, in section 3, we critically assess their interpretation. Our main focus in this section is on the meaning of information in their approach to verify that to what extent (if any) they could coherently explain the peculiarities of quantum world. Subsequently, in section 4, we will show that once one is going to interpret Brukner and Zeilinger's measure of information in a real extension of the spin measurement, it cannot quantify the amount of uncertainty in a suitable manner.

\section{Elements of Brukner and Zeilinger's Interpreta- tion of Quantum Mechanics}

The first topic in Brukner and Zeilinger's interpretation is that the whole physical description is based on propositions. Application and selection of propositions is not arbitrary in their attitude; rather it depends on what we want to learn about Nature and what knowledge about Nature we intend to discuss with others ${ }^{2}$. Emphasizing the role of propositions in physical description, Brukner and Zeilinger persist in a fundamental point which is a basic element in their interpretation: A quantum system is a construct based on complete list of propositions together with their truth values. A proposition, they assert for example, could be (1) "The velocity of the object is $v$ " or (2) "The position of the object is $x$ ". But since in quantum mechanics it is usually impossible to determine simultaneously the truth values of two arbitrary propositions, if the proposition (1) is definite, then the proposition (2) is completely indefinite and vice versa. The two propositions, here, are mutually exclusive. Brukner and Zeilinger consider this as a special case of quantum complementarity. In this way, a new language is represented for the physical description of quantum formalism, a language in which the propositions construct the essence of quantum systems instead of merely describing them. This is a starting point for critical arguments about their interpretation.

\footnotetext{
${ }^{2}$ This can be interpreted as an indication of intentionality in the transmission of information which demands a semantic concept of information. We will talk more about this point in next section.
} 
The language of propositions in quantum mechanics is similar to the language which is used for the description of classical systems, except for the complementary situations which must also be taken into account in the quantum description. While we can always assign definite truth values to all propositions in (deterministic) classical theories, one cannot assign simultaneously definite truth-values to mutually exclusive propositions in quantum mechanics. But even in cases that the truth values of two mutually exclusive propositions cannot be assigned simultaneously, we can always specify the amount of information about them. So, according to Brukner and Zeilinger, this reveals a more fundamental notion which is knowledge and information: We can quantify our information about descriptive propositions in both the classical and quantum domains. What is changed, however, in passing from classical to quantum world is the replacement of the definite truth values with the finite amounts of information. They believe that this is the least expense which could be paid for the change in the epistemological structure of classical physics. In this view, information is regarded as the most fundamental notion [8]:

"In contrast [with Bell's idea] it is suggested that information is the most basic notion of quantum mechanics, and it is information about possible measurement results that is represented in the quantum states." 3

We believe, however, the expense is high, since this expense is not merely paid for substituting the non-deterministic finite information in quantum mechanics in place of the deterministic complete description of classical systems. It is also paid for introducing the finiteness of information as an indispensable ingredient of a quantum object itself. What is replaced here, is the information content of the systems, not merely the amount of available information about the systems [4]:

"The information content of a quantum system is finite.

With this we mean that a quantum system cannot carry enough information to provide definite answers to all questions that could be asked experimentally. Then, by necessity the answer of the quantum system to some questions must contain an element of randomness. This kind of randomness must then be irreducible, that is, it cannot be reduced to 'hidden' properties of the system. Otherwise the system would carry more information than what is available."

Subsequently, Brukner and Zeilinger conclude that irreducible randomness results from the finiteness of information [4]:

\footnotetext{
${ }^{3}$ Emphases in all quotations throughout this text are original.
} 
"Thus, without any additional physical structure assumed, we let the irreducible randomness of an individual event and complementarity [both], be a consequence of the finiteness of information."

In response to the question that how much information a quantum system can carry, they introduce the principle of quantization of information for an elementary system. According to this principle, a descriptive proposition for a complex system can be subdivided to constituent propositions until we reach a final limit. The individual system that represents the truth value of one single proposition only, is called elementary system. Beyond this limit, information is irreducible. So the principle of quantization of information states that [1]:

"An elementary system carries 1 bit of information."

For a complex system, consisting of $N$ elementary systems this principle is generalized to [1]:

" $N$ elementary systems carry $N$ bits."

The principle of quantization of information does not make any statement about how the $N$ bits of information are distributed over the $N$ systems. Brukner and Zeilinger consider this principle for systems with both independent and entangled subsystems.

For example, for a spin- $\frac{1}{2}$ system (like an electron) there are always three complementary or mutually exclusive propositions for describing the spin situation. These are: "The spin along $\overrightarrow{n_{1}}$ is up (down)", "The spin along $\overrightarrow{n_{2}}$ is up (down)" and "The spin along $\overrightarrow{n_{3}}$ is up (down)", where $\overrightarrow{n_{1}}$, $\overrightarrow{n_{2}}$ and $\overrightarrow{n_{3}}$ are mutually orthogonal directions. Complete information about one of the propositions is possible only through complete ignorance of two others. Also for any other two-valued observable, there are three complementary propositions for describing the state of system, even though these propositions are not related with three space directions.

Now this question arises as to how the amount of information (or the amount of uncertainty) could be quantified in a statistical prediction? Brukner and Zeilinger remark that in classical measurements in which it is assumed that the classical system has predetermined physical properties before a measurement is performed, the Shannon information is an appropriate measure of uncertainty which can be defined as the following relation for $n$ possible outcomes:

$$
H(\vec{p})=-\sum_{i=1}^{n} p_{i} \log p_{i}
$$


where $\vec{p}$ is a probability vector in the probability space with length $|\vec{p}|=$ $\sqrt{\sum_{i=1}^{n} p_{i}^{2}}$ in which $p_{i}$ is the probability of occurrence of the outcome $i$ in a given measurement. For quantum measurements, however, they introduce a new measure of information which is defined as $[2,3]$ :

$$
I(\vec{p})=\mathcal{N} \sum_{i=1}^{n}\left(p_{i}-\frac{1}{n}\right)^{2}
$$

where $\mathcal{N}$ is a normalization factor. In general, for a system in which maximally $k$ bits of information can be encoded, $n=2^{k}$ and $\mathcal{N}=2^{k} k /\left(2^{k}-1\right)$. For an elementary system which can carry only one bit of information, $n=2$. Then, one can reach the following relation:

$$
I\left(p_{1}, p_{2}\right)=2\left(p_{1}-\frac{1}{2}\right)^{2}+2\left(p_{2}-\frac{1}{2}\right)^{2}=\left(p_{1}-p_{2}\right)^{2}
$$

where $p_{1}$ and $p_{2}$ are the probabilities defined for a dichotomic observable and $p_{1}+p_{2}=1$. If one of the probabilities is one, $I$ reaches its maximal value of 1 bit of information which is equivalent to the complete certainty. If both probabilities are equal, $I$ takes its minimal value of 0 bits of information which implies a complete uncertain (or a complete random) situation.

We can write the measure of information (3) as $I=i^{2}$ where $i=p_{1}-p_{2}$. Moreover, one can generalize the relation (3), if the vector $\vec{i}$ is defined in the information space as $\vec{i}=\left(i_{1}, i_{2}, i_{3}\right)=\left(p_{x}^{+}-p_{x}^{-}, p_{y}^{+}-p_{y}^{-}, p_{z}^{+}-p_{z}^{-}\right)$, where, e.g., $p_{z}^{+}$denotes the probability of finding the spin-up result along the $z$ direction. Vector $\vec{i}$ characterizes the information state of an elementary system which is, in Schrödinger's terminology, a catalog of knowledge about a set of three mutually complementary propositions [4]:

"It is assumed that the catalog $\vec{i}$ is a complete description of the system in the sense that its knowledge is sufficient to determine the probabilities for the outcomes of all possible future measurements."

Then, the quantum state is also considered as the catalog of knowledge. According to the measure of information indicated in relation (3), the total information of an elementary system is defined as a sum of the individual measures of information over a complete set of mutually complementary propositions

$$
I_{\text {total }}=I_{1}+I_{2}+I_{3}=1
$$

where, $I_{1}=i_{1}^{2}, I_{2}=i_{2}^{2}$ and $I_{3}=i_{3}^{2}$. Since an elementary system carries 1 bit of information, the total information is also equal to one. So, in different 
experimental setups where the components of $\vec{i}$ adopt different values, the total information is always constant and equal to one for pure states.

In Brukner and Zeilinger's interpretation, an entangled state is also important from this point of view that an entangled system has more information than its individual constituents $[1,4,5]$. For example, consider the following singlet state for a pair of spin-half particles which is known as one of the four entangled Bell states:

$$
\left|\Psi^{-}\right\rangle=\frac{1}{\sqrt{2}}\left(|\widehat{n},+\rangle_{1}|\widehat{n},-\rangle_{2}-|\widehat{n},-\rangle_{1}|\widehat{n},+\rangle_{2}\right)
$$

where $|\widehat{n},+\rangle_{1}$, e.g., is the spin-up state of particle 1 along $\widehat{n}$-direction (which can be chosen arbitrary). The total information represented by the entangled state $(5)$ is $I_{C o r r}^{B e l l}=2$. This means that two bits of information are carried by the whole system, corresponding to statements about the results of joint observations, "The two spins are the same along $x$ " and "The two spins are the same along $y$ ". The truth values of these propositions is falsefalse. Since any product state of two particles (like each single term in relation (5)) carries only one bit of information, for any entangled state we have $I_{\text {Corr }}^{\text {entgl }}>1^{4}$. This is an important information condition which is the characteristic feature of entangled states. Entanglement here is believed to be a secondary concept which its advantage is only due to its information content.

In this interpretation, wave function is only a mathematical representation which contains encoded information about a quantum system. Our knowledge about a system gives it the possibility of being in a place at a given moment. In the measurement process, the quantum state which represents that knowledge changes. This change is instantaneous, but it is only a change in our knowledge. Our information about all of the other spatial points also changes instantaneously. However, there is nothing to be transmitted in a physical manner: There is no faster than light signalling. In addition, the total information content of the system remains constant [4]:

"Unlike a classical measurement, a quantum measurement thus does not just add (if any) some knowledge, it changes our knowledge in agreement with a fundamental finiteness of the total information content of the system."

The total information content of a system, $I_{\text {total }}$, is invariant under the change of the representation of the catalog $\vec{i}$ and remains constant under any rotation in the information space. Brukner and Zeilinger determine the rotation matrices for Euler angles, with assumptions: (1) The invariance of

\footnotetext{
${ }^{4} I_{C o r r}^{\text {entgl }}$ can be calculated for any set of spin measurements of two particles along two arbitrary directions. For more details, see for example [7].
} 
the total information under rotation and (2) the homogeneity of rotational angles (in the sense that adding a constant value to any of three rotational directions, does not change the physics of the problem and the location of vector $\vec{i}$ in the information space remains unchanged) [4]. According to these two assumptions, they show that for a spin-half particle, angular momentum generates the rotation. Correspondingly, they want to extend a new formulation of quantum mechanics which is constructed on the information space, instead of an abstract vector space. Moreover, they show that the following dynamical equation in quantum mechanics,

$$
i \hbar \frac{d \widehat{\rho}(t)}{d t}=[\widehat{H}(t), \widehat{\rho}(t)]
$$

can be derived for an elementary system from the time evolution of catalog of knowledge $\vec{i}$, in the information space [4]. In relation (6), $\widehat{\rho}(t)$ and $\widehat{H}(t)$ are the density matrix and the Hamiltonian of system, respectively. A key assumption here is that, for an isolated system with no information exchange with the environment, the total information of system is conserved in time. I.e.,

$$
I_{\text {total }}(t)=\sum_{n=1}^{3} i_{n}^{2}(t)=\sum_{n=1}^{3} i_{n}^{2}\left(t_{0}\right)=I_{\text {total }}\left(t_{0}\right)
$$

There are still unsolved problems which are to be solved, as mentioned by themselves too. The measure of information defined in relation (2) has not yet been studied for continuous variables. One should also elucidate that in the information space, e.g., how momentum can be inferred as the generator of transformation and how the Schrödinger equation can be reformulated consistently. There is also no complete set of complementary observables for infinite Hilbert spaces ${ }^{5}$.

To sum up, the ultimate goal in Brukner and Zeilinger approach is to show that information is a fundamental notion which not only the inherent nature of quantum particles and quantum processes are based on its concept, but also a new formulation can emerge from its foundation. External reality depends as well on our experimental answers to questions about the quantum events [4]:

"Therefore the experience of the ultimate experimenter is a stream of ('yes' or 'no') answers to the questions posed to Nature. Any concept of an existing reality is then a mental construction based on these answers. Of course this does not imply that reality is no more than a pure subjective human construct. From our observations we are able to build up objects with a set of properties that do not change under variations of modes of observation or

\footnotetext{
${ }^{5}$ We are grateful to anonymous referee for reminding this point.
} 
description. These are 'invariants' with respect to these variations."

Note that in the above expression, what can be predicted by an observer is a qualitative picture of physical properties. This point has been indicated explicitly by Zeilinger elsewhere [8]:

"[T]he observer has a qualitative, but not a quantitative influence on reality. She can define which quality will show up in the experiment, but not the quantity, the exact value, the latter being completely random, except in the rare case when the quantum system is in an eigenstate of the observed quantity."

By this, Zeilinger means that for example when an observer wants to measure the energy of a system, she knows that with her measurement the property of energy will appear. She can define energy as a qualitative notion, but its exact value is not predictable or definable.

Meanwhile, the randomness of the events arises from the fact that information is finite [4]:

"It is beyond the scope of quantum physics to answer the question why events happen at all (that is, why the detectors clicks at all). Yet, if events happen, then they must happen randomly. The reason is the finiteness of the information."

Even the Bohr complementarity is regarded as a consequence of the finiteness of information [8]:

"Complementarity then simply is a consequence of the fact that the total information which is represented by a quantum system is finite."

\section{Comments On The Information Concept}

In reformulating the quantum dynamical equation (6), two basic assumptions were considered: 1) The total information is a conserved quantity and 2 ) the components of the information vector generates $\mathrm{SU}(2)$. Second assumption says that the time evolution of the information vector is unitary. Since in this interpretation, we are presented with the variation of the information vector together with the invariance of the total information in the measurement process too, this important question appears as to why it is not defined any time evolution for the information vector during the measurement. Note that this question comes within the scope of the interpretation itself and does not refer to subjects like the objectivity of the quantum system. So, it seems that there are two kinds of information debated here: 
Information before measurement and information after measurement. The time evolution of information before measurement is unitary and reversible with time, but information during measurement evolves irreversibly: We can always reproduce the initial information from the information given at a later time (and vice versa) except for the case that a measurement is performed in the intervening time. Why should the information vector evolve in different ways? What is the concept of instantaneous change in information that occurs during the measurement? Does information really have a dual nature? How does the finiteness of information explain this duality? If information is a fundamental concept, in what manner can one realize the distinction in the basis?

On the other hand, many people think that quantum mechanics must agree with classical mechanics in a suitable limit. So, if quantum dynamics could be constructed on an underlying definition of information, it would be expected that in such an approach, the new measure of information could become consistent with a classical description in an appropriate limit too. Accordingly, what is the relation between the classical and the quantum worlds as far as the notion of information is concerned? And considering Brukner and Zeilinger's new measure of information, how can one explain the apparent consistency of the two worlds in Nature?

In this regard, Timpson distinguishes two points of view in Brukner and Zeilinger interpretation: an instrumentalism versus a phenomenalism $[12,13]$. In this interpretation, a quantum state merely describes the probabilities of the possible measurement results, a way for representing the outcomes of all possible future observations [1]. This characterizes their instrumentalist point of view. On the other hand one can discern a form of phenomenalism according to which physical objects are taken to be not an actual things, but to be some constructs relating observations. So, as Timpson states [12],

"[A] system represents a quantity of information about measurement results because a physical system literally is nothing more than an agglomeration of actual and possible sense impressions arising from observations."

As a consequence of the combination of these two attitudes, Timpson argues that the principle of quantization of information cannot function as a foundational principle for quantum mechanics, nor does it have the explanatory power that Brukner and Zeilinger suppose [12, 13].

It is, however, important to remember that all features of this interpretation cannot be categorized as instrumentalist or phenomenalist perspectives. As an instance, one can mention the very meaning of information here which is introduced too fundamentally to be understood as a form of phenomenalism or instrumentalism. Nonetheless, one sees no clear definition of 
information and its conception in this interpretation. (A detailed discussion of this subject is given in Appendix.)

The principle of finiteness of information is also a matter of controversy. Indeed, how can one verify that a quantum system must essentially have a finite information content? Many people may still be interested to know why we should have such a constraint about the possible measurement results. As many people, for example, are still curious to know why the thermodynamic systems inherently tend to go towards most probable states according to the Second Law of Thermodynamics. Since, one may some day discover a thermodynamic force which will explain the underlying reason of this tendency.

Yet, there are some people who believe that an unlimited amount of information can be coded in a given quantum state. This can precisely be illustrated by Wiesner's quantum multiplexing argument which states that one can always code two distinct one-bit messages into a spin- $\frac{1}{2}$ system [17]. However, the spin state is not an observable and there is no accessible information larger than a single bit. No observer can read both bits, but it is only possible to read one bit of information in any proper choice of measurement. The finiteness of gaining knowledge about the properties of a system is not a specific feature of quantum mechanics only. Information constraints also exist for classical systems [18]. On the other hand, any argument about the finiteness of information is contingent upon the fact that how one interprets the probability, not upon the fact that how one determines the quantity of information.

Brukner and Zeilinger consider the randomness as an intrinsic property for quantum systems. But, they conclude this not as a possible interpretation of probability coming from outside the theory. Instead, they believe that this is a natural consequence of the principle of finiteness of information. But, as Markus Arndt has recently noted [19]:

"Objective (absolute) randomness is hardly fully verifiable. One may exclude certain classes of causes / reasons for a quantum choice ........ But the a priori exclusion of any reason whatsoever cannot be falsified / verified."

Brukner and Zeilinger try to show that everything, even an objective event which happens in an experiment results from the finiteness of information. This point is expressed explicitly in the abstract of one of their papers in which they discuss conceptually the double-slit experiment for $C_{60}$ molecules [6]:

"It is argued here that quantum interference is a consequence of the finiteness of information." 
This attitude originates again from the fact that information plays a central role in quantum world. So, they attempt to demonstrate that objective properties of quantum systems which appear in measurements are merely informational effects. Subsequently, they conclude that the interference pattern of quantum particles is an objective consequence of the finiteness of information. In other respects, information loses its fundamental role and the question that how the interference pattern is formed (or any other objective property that appears in a measurement) remains without answer.

Nevertheless, what really they show is that:

1) there exists a limitation in observing both the path and the interference pattern of a quantum particle simultaneously and,

2) this limitation is a consequence of the finiteness of information content of a quantum system.

In other words, what they show is that we should not expect we could obtain a desirable classical knowledge about a quantum object. Logically, however, this does not mean that:

$\left.2^{\prime}\right)$ the interference too, is a consequence of our constraints in acquiring the information.

There is a clear distinction in the meaning of statements 2 and $2^{\prime}$. The statement $2^{\prime}$ cannot be deduced naturally from their discussion, but (it seems) it relies primarily on their phenomenalistic position about the microobjects. This point of view is not of course compelling. But, even if we adopt this view, we cannot still conclude that the interference pattern is a result of the finiteness of information too. The finiteness of information does not explain how these objective events appear, even though we put it into the ontology of quantum objects.

At the end, it must be pointed out that some kind of incongruity is realized in their interpretation. In discussing the objective reality and its relation with information, at first sight, Zeilinger takes these notions on the same footing, so that neither one is sufficient for understanding the quantum world [1]:

"Therefore, while in a classical worldview, reality is a primary concept prior to and independent of observation with all its properties, in the emerging view of quantum mechanics the notions of reality and of information are on an equal footing. One implies the other and neither one is sufficient to obtain a complete understanding of the world." 
But after that, Brukner and Zeilinger take stronger position and consider the physical properties of objects as secondary and information as primary notions. In other words, a real property is viewed as a representation of information which is created in measurement [4]:

"In classical physics a property of a system is a primary concept prior to and independent of observation and information is a secondary concept which measures our ignorance about properties of the system. In contrast in quantum physics the notion of the total information of the system emerges as a primary concept, independent of the particular complete set of complementary experimental procedures the observer might choose, and a property becomes a secondary concept, a specific representation of the information of the system that is created spontaneously in the measurement itself."

There is no further explanation that why the reality of physical properties becomes suddenly a secondary concept. Also, it is not obvious how reality is created spontaneously in the measurement.

\section{Critical Assessment of Brukner and Zeilinger's measure of information}

Brukner and Zeilinger's definition of a measure of information is derived initially from an uncertainty expression for a specific outcome in $N$ trials of an experiment. This can be defined as $\frac{\sigma^{2}}{N}=p(1-p)$ where $p$ is the probability of the occurrence of a dichotomic result and $\sigma^{2}$ is variance for binomial distribution $[2,4]$. Then, for $n$ outcomes with the probabilities $\vec{p} \equiv\left(p_{1}, p_{2}, \ldots, p_{n}\right)$ of the individual occurrences, they deduce the relation (2) as an appropriate measure of information.

A crucial point here is that their measure of information requires not only that probabilities are known, but also that the number of possible outcomes $n$ is known (see relation $(2))^{6}$. In turn, the value of $n$ is determined by $k$ which is defined as the maximum bits of information that can be encoded in a quantum system $[2,4]$. As a matter of fact, this means that two different notions of total information are to be taken into account in Brukner and Zeilinger's interpretation:

1) A total information $k$ defined as an input of the relation (2) which is determined by knowing the number of possible outcomes $n$,

2) A total information $I_{t o t}$ which is an output of relation (2) as the sum of the individual measures of information.

\footnotetext{
${ }^{6}$ We are grateful to anonymous referee for emphasizing this point.
} 
Considering those cases where $k$ bits of information can be maximally encoded, Brukner and Zeilinger define $n=2^{k}$, or $k=\log _{2} n$ [2]. But, where is basically $k$ coming from? The answer is that if one supposes $n$ equiprobable events which each event could occur with the same probability $\frac{1}{n}, k$ would be precisely the Shannon information $H(\vec{p})$ for $p_{i}=\frac{1}{n}$. Here, $k$ is essentially endowed with a counterfactual meaning of information in the Shannon sense of definition. One may consequently interpret that Brukner and Zeilinger's measure of information in (2) does need a form of the socalled microcanonical Shannon entropy as an input. So, $k$ and $I_{t o t}$ are not principally the same and it is expected that they should not be the same numerically in some circumstances too.

To elaborate this point more palpably, let us consider that in a real experiment, there will be a non zero probability of non-detection for measuring each spin component of a spin half particle. Suppose also that, ideally, the spin of particle would be expected to be up along $x$. Then, if the overall efficiencies of the measuring apparatuses are assumed to be the same along different directions, one can describe the statistics of the three possible events of spin up detection $(+)$, spin down detection $(-)$ and no detection (0) along $x, y$ and $z$ with the following probabilities, respectively:

$$
\begin{aligned}
& p_{x}^{+}=\eta, p_{x}^{-}=0, p_{x}^{0}=1-\eta \\
& p_{y}^{+}=p_{z}^{+}=\frac{1}{2} \eta, p_{y}^{-}=p_{z}^{-}=\frac{1}{2} \eta, p_{y}^{0}=p_{z}^{0}=1-\eta
\end{aligned}
$$

where $\eta$ denotes the overall efficiency; $p_{x}^{0}$, e.g., is the probability of nondetection along $x$ and the detection probabilities are defined as before. Using the relation (2), one can obtain the individual information functions $I_{1}, I_{2}$ and $I_{3}$ along $x, y$ and $z$, respectively, for $n=3$ and $k=\log _{2} 3$ :

$$
\begin{aligned}
& I_{1}=\frac{3 \log _{2} 3}{2}\left[\left(\eta-\frac{1}{3}\right)^{2}+\left(\eta-\frac{2}{3}\right)^{2}+\frac{1}{9}\right] \\
& I_{2}=I_{3}=\frac{3 \log _{2} 3}{2}\left[\frac{1}{2}\left(\eta-\frac{2}{3}\right)^{2}+\left(\eta-\frac{2}{3}\right)^{2}\right]
\end{aligned}
$$

After some simple algebra, one can get

$$
I_{\text {total }}=I_{1}+I_{2}+I_{3}=\frac{3 \log _{2} 3}{2}\left(5 \eta^{2}-6 \eta+2\right)
$$

The ratio of $\frac{I_{\text {total }}}{k}$ plotted as a function of $\eta$ is shown in figure1. For $\eta \rightarrow 1$ (perfect efficiency), the number of possible outcomes $n$ reduces to 2 (i.e., there is no non-detection event) and the relation (3) will be the 
appropriate expression for each individual measure of information. Then, $I_{\text {total }}=k=1$ describes the total information content of an elementary system (in our example, a spin half particle), according to Brukner and Zeilinger's interpretation. However, for $0<\eta<1$, there are three possible outcomes and while in such an experiment $k$ denotes that a value of $\log _{2} 3 \approx$ 1.585 bits of information can be maximally encoded (according to their definition), $I_{\text {total }}$ is given by (10) which depends on the efficiency factors of measuring devices. Since, in different experiments $\eta$ may not be the same, $I_{\text {total }}$ cannot be considered as a conserved value in principle. Specially, for $0<\eta<0.29$ and $0.91<\eta<1, I_{\text {total }}$ exceeds the maximal possible amount of information, i.e., 1.585 bits that can be encoded in an individual spin half particle. This is in contrast to what is expected for $I_{\text {total }}$ in the Brukner and Zeilinger's sense of definition [3]:

"Independent of the various possibilities to encode information, the total information content of the system cannot fundamentally exceed the maximal possible amount of information that can be encoded in an individual observable."

In contrast, Shannon information calls only for a knowledge of probabilities and its change is isomorphic for different $n$. Its description is also clear for the above example. Let us define $H_{x}, H_{y}$ and $H_{z}$ as the corresponding Shannon uncertainties along the directions $x, y$ and $z$, respectively. Then, one can obtain:

$$
\begin{aligned}
H_{x} & =-\eta \log _{2} \eta-(1-\eta) \log _{2}(1-\eta) ; \\
H_{y} & =H_{z}=-\eta \log _{2} \frac{\eta}{2}-(1-\eta) \log _{2}(1-\eta) \\
& =H_{x}+\eta
\end{aligned}
$$

While for $\eta \rightarrow 1, H_{x}$ indicates that there is no uncertainty about the value of the spin component of particle along $x$, it increases as $\eta$ is being reduced to $\frac{1}{2}$. This is the point where we have the maximum uncertainty of one bit for the spin component along $x$. Moreover, $H_{y}\left(=H_{z}\right)$ reaches its maximum value of $\log _{2} 3 \approx 1.585$ bits (which is the same value of $k$ ) for $\eta=\frac{2}{3}$. Nevertheless, as $\eta$ approaches to zero, all the uncertainties $H_{x}, H_{y}$ and $H_{z}$ decrease, because the probabilities of detection along different directions approaches to zero. The uncertainties $H_{x}$ and $H_{y}\left(=H_{z}\right)$ are plotted against $\eta$ in figure 2.

The above example shows that the Shannon and the Brukner-Zeilinger measures of information may have very different descriptions even for simple cases, when merely the primary definitions are extended to more concrete circumstances. It casts doubt upon the fact that whether the latter measure of information can retain its significance for more realizable physical examples. 


\section{Conclusions}

Brukner and Zeilinger's interpretation has brought up some provoking topics. Statements about the essential nature of information, the finiteness of the information content of a quantum object, the possibility of deriving the quantum dynamics from an information basis and the definition of a new measure of information, all are interesting subjects which call for careful analysis. Considering information as a fundamental notion, however, this interpretation encounters some difficulties and leaves us alone with not answered basic questions. It is not clear why a quantum object should have a finite information content in its essence, how the information vector evolve when one measures a physical quantity, and what really means as an instantaneous change in information during the measurement. Here, we are confronted with a complex, multidimensional notion of information which its foundation is not clear.

On the other hand, Brukner and Zeilinger's definition of a new measure of information may lose its significance, when an elementary system is treated realistically in the spin measurements. Here, the possible number of outcomes $n$ may discriminate the value of the total information content in ideal and non-ideal measurements. So, one is encountered this basic question that what does an elementary system actually mean, when its information content cannot be conserved in real practice?

Acknowledgment. A. Shafiee acknowledges Atomic Energy Organization of Iran (AEOI) for financial support. The authors also thank anonymous referee of this journal for many helpful comments.

\section{Appendix}

Information is a notion with different concepts, and each concept has its own application(s) $[20,21,22]$. It is hardly possible that a single concept of information can account for the various applications of this extensive field. However, it is worth looking into a special notion of information here: Information with semantic concept is something capable of providing knowledge [20]. In Routledge Encyclopedia of Philosophy, one reads [23] "The term information has various senses in ordinary discourse, including knowledge ..... and propositional content ..... Information in these senses has semantic features, such as reference and truth-value."

The main aspect of semantic information is about what a message or proposition means. In this sense, knowledge and semantic information are relative notions. To have knowledge about a system, we should describe the information content of that system by using the descriptive propositions. Once information is available, knowledge can be built in terms of justified information.

As stated by Dretske in his semantic theory of information (see [24], p. 45): "A state of affairs contains information about $\mathrm{X}$ to just that ex- 
tent to which a suitable placed observer could learn something about X by consulting it" (Quoted also in [20]). Dretske also qualifies information as a semantic concept in virtue of the intentionality inherent in its transmission. Information receives its intentional character from the lawful regularities on which it depends (for example, the laws of physics in the domain of physics). Yet, it is not relevant in this view to ask where the intentional character of laws comes from (see [24], p. 77 and also [20]). This makes some conceptual difficulties regarding a well-defined relation between information and the physics from which information stems. Floridi also analyzes the meaning of semantic information in terms of well-formed, meaningful and truthful data with a propositional orientation [22]. Here, "well-formed" means that data have been put together correctly according to some given rules and "meaningful" means that the data must also comply with the meanings of propositions. So, the semantic content in his view is formed on the basis of the well-formed and meaningful data represented by propositions with definite truth values which in turn are used to talk about the world and describe it.

On the other hand, as Shannon himself remarks [9]:

"[S]emantic aspects of communication are irrelevant to the engineering problem. The significant aspect is that the actual message is one selected from a set of possible messages."

The Shannon measure of information is purely quantitative. It only deals with amounts of information and disregards questions related to information content or the meaning of information. Shannon information is commonly described as a study of information at the syntactic level, which can be studied by the Mathematical Theory of Communication, also known as Communication Theory or Information Theory. According to syntactic concept, information theory is introduced with the application of random variables and probability distributions, so it is a mathematical theory. It has applicability to a variety of fields like communication theory, thermodynamics, computer science and algorithmic complexity [20].

The concept of information can also be treated physically. By the term physical, we mean that information either can be transmitted via a physical entity (i.e., a signal), or is an objective property of the system which can be described by the physical laws, or both. According to this view, information is transmitted from one point to another point via a physical entity, i.e., an information-bearing signal. This is in opposition to a semantic view where states that to establish an informational link between two distant locations, it is only necessary that one can have a knowledge at one location by looking at the other one. This point is similar to what is declared by Brukner and Zeilinger in one of their articles [4]:

"When the state of a quantum system has a non-zero value at 
some position in space at some particular time, it does not mean that the system is physically present at that point, but only that our knowledge (or lack of knowledge) of the system allows the particle the possibility of being present at that point at that instant."

There is no direct reference to a specific notion of information in Brukner and Zeilinger's interpretation. Nonetheless, it seems that the meaning of information has a complex character in their approach. On one side, it appears that a semantic character for information is more justified here. For a given system, measure of information implies what can be said quantitatively about possible measurement results by assigning truth values to corresponding descriptive propositions. The quantum state also represents the possible ways one can assign truth values to these propositions.

On the other side, information is treated as the base for quantum physics in this interpretation. So, how can one reformulate the quantum dynamics with a notion of information which seems to be not physical in its essence? Hence, the question appears as to whether quantum physics is reducible to an information modeling. Could the universe primarily be made of information? And if so, how does matter emerge from information?

\section{References}

[1] A. Zeilinger, Found. Phys. 29, 631 (1999).

[2] Č. Brukner and A. Zeilinger, Phys. Rev. Lett. 83, 3354 (1999).

[3] Č. Brukner and A. Zeilinger, Phys. Rev. A 63, 022113 (2001).

[4] Č. Brukner and A. Zeilinger, e-print arXivPreprint quant-ph/0212084

[5] Č. Brukner, M. Żukowski and A. Zeilinger, e-print arXiv:quant-ph/0106119.

[6] Č. Brukner and A. Zeilinger, Phil. Trans. R. Soc. Lond. A 360, 1061 (2002), e-print arXiv:quant-ph/0201026.

[7] Č. Brukner, M. Aspelmeyer and A. Zeilinger, e-print arXiv:quant-ph/0405036.

[8] A. Zeilinger: "Bell's Theorem, Information and Quantum Physics". In: Quantum [Un]speakables, From Bell to Quantum Information, edited by R. A. Bertlmann and A. Zeilinger (Springer-Verlag, Berlin, 2002), pp. 241-254.

[9] C. E. Shannon, Bell Syst. Tech. J. 27, 379 (1948). 
[10] J. Řeháček and Z. Hradil, e-print arXiv:quant-ph/0111115.

[11] E. F. Galvão, e-print arXiv:quant-ph/0212124.

[12] C. G. Timpson, Stud. Hist. Phil. Mod. Phys. 34, 441 (2003).

[13] C. G. Timpson, The applicability of the Shannon information in quantum mechanics and Zeilinger's Foundational Principle. PSA 2002 Contributed Papers, PITT-PHIL-SCI-00000710.

[14] A. Duwell, Stud. Hist. Phil. Mod. Phys. 34, 479 (2003).

[15] M. J. W. Hall, e-print arXiv:quant-ph/0007116.

[16] P. G. L. Mana, Phys. Rev. A 69, 062108 (2004), e-print arXiv:quant-ph/0302049.

[17] B. Schumacher: "Information from Quantum Measurements". In: Complexity, Entropy And The Physics Of Information, Santa Fe Institute Studies in the Sciences of Complexity No. VIII, edited by W. H. Zurek (Addison-Wesley, Redwood City, 1990), pp. 29-37.

[18] L. Brillouin: Scientific Uncertainty, and Information, (Academic Press, New York, 1964), Part II.

[19] M. Arndt et al, e-print arXivPreprint quant-ph/0505187.

[20] O. Lombardi, Found. Sci. 9, 105 (2004).

[21] M. Horodecki, R. Horodecki, A. Sen(De) and U. Sen, e-print arXiv:quant-ph/0407038

[22] L. Floridi, Metaphilosophy, 35, 554 (2004).

[23] K. M. Sayre, "Information Theory". In: Routledge Encyclopedia of Philosophy, Version 1.0, (Routledge, London and New York,1998).

[24] F. I. Dretske: Knowledge and the Flow of Information, (MIT Press, Cambridge, 1981). 
Figure 1: $\frac{I_{\text {total }}}{k}$ is plotted as a function of $\eta$. The minimum occurs at $\eta=0.6$, where $\frac{I_{\text {total }}}{k}=0.3$.

Figure 2: The Shannon uncertainties $H_{x}$ and $H_{y}\left(=H_{z}\right)$ are plotted against $\eta$. The maximums of uncertainty are one bit for $H_{x}$ at $\eta=0.5$ and $\log _{2} 3 \approx$ 1.585 bits for $H_{y}\left(=H_{z}\right)$ at $\eta=\frac{2}{3}$. 
This figure "BZfig1.JPG" is available in "JPG" format from: http://arxiv.org/ps/quant-ph/0407198v2 
This figure "BZfig2.JPG" is available in "JPG" format from: http://arxiv.org/ps/quant-ph/0407198v2 